\title{
Secure Network Coding with Erasures and Feedback
}

\author{
László Czap \\ EPFL, Switzerland \\ laszlo.czap@epfl.ch
}

\author{
Christina Fragouli \\ EPFL, Switzerland \\ UCLA, USA \\ christina.fragouli@epfl.ch
}

\author{
Vinod M. Prabhakaran \\ TIFR, India \\ vinodmp@tifr.res.in
}

\author{
Suhas Diggavi \\ UCLA, USA \\ suhas@ee.ucla.edu
}

\begin{abstract}
Secure network coding assumes that the underlying network links are lossless, thus it can be applied over lossy networks after channel error correction. Yet it is well known that channel losses, such as packet erasures, can be constructively used for secrecy over a link. We address here the challenge of extending these results for arbitrary networks. We provide achievability schemes over erasure networks with feedback, that outperform the alternative approach of channel error correction followed by secure message transmission separation. We derive outer bounds on the securely achievable rate and as a consequence we show optimality of our proposed scheme in some special cases.
\end{abstract}

\section{INTRODUCTION}

Secure network coding assumes that the underlying network links are lossless; thus if a wiretapper, Eve, observes a link, she gets access to all the information that flows through it. Since most practical channels introduce errors, underlying the lossless assumption is an implicit "channel coding" followed by "security coding" separation: if our links introduce errors, we need first apply a channel code to correct them, and then build security on top of the resulting lossless networks. But as a result, we can convey zero rate securely through the links that Eve observes.

It is well known that channel losses, such as erasures, can be constructively used to enable non-zero secrecy rate over a link. Assume for example that Eve observes a node's transmission independently and with a larger erasure probability than the legitimate next hop node; then, by applying a wiretap code [1] we can convey through this link a message at a nonzero rate. Moreover, if we allow channel state feedback, i.e., the next hop node to acknowledge packet reception as is the case in most network protocols today, we can convey a nonzero rate even if the eavesdropper has a better channel than the legitimate receiver [2].

The challenge is how to extend these results from single links to arbitrary networks. For a single link, we have an exact characterization of the secret message capacity with feedback, yet as soon as we go to a network with more than one hop and multiple nodes, the complexity of the problem increases exponentially, as there exists an exponential number of subsets of nodes that can generate randomness, create secret keys, and cooperate for secrecy. Finding secrecy

This work was supported by the ERC Starting Grant Project NOWIRE ERC-2009-StG-240317. V. M. Prabhakaran's research was supported in part by a Ramanujan Fellowship from the Department of Science and Technology, Government of India. The work of S. Diggavi was supported in part by NSF award 1136174 and MURI award AFOSR FA9550-09-064. capacity of a general network is as hard as determining the capacity region of multiple unicast network coding, which is a long-standing open problem [3], [4].

This paper provides achievability schemes over arbitrary erasure networks with link-by-link state feedback. We start from the simplest case, independent erasure networks with the same erasure probability $\delta$ in each link. We assume that there exist $h$ edge-disjoint paths from the source to each receiver, and that our eavesdropper, Eve, observes any $z$ links in the network. When eavesdropping transmissions on a link that connects node $u$ to node $v$, Eve also receives the transmissions of node $u$ with erasure probability $\delta_{E}$, independently from node $v$. We also make the assumption that only the source node can generate randomness; this assumption clearly reduces the rates we can achieve, but at the same time simplifies the problem, and fits well with the current networking philosophy of having the intelligence at the edge of the network and keeping intermediate node operations simple.

Our proposed scheme can achieve secrecy rates that consistently outperform a separate channel error correction and secure message transmission approach. Our scheme applies a separation of two phases, where at a first stage we generate shared randomness (key) between the source and the receivers, we use the key for encryption and at the second phase we reliably send the encrypted message. This approach is known to achieve secrecy capacity over a single link [2].

We first consider lossless networks and establish connection between the secure network coding scheme [5], [6] and the two phase approach. We propose a modified, two phase secure network coding scheme showing that there is no fundamental difference between these approaches.

We then examine how we can take advantage of erasures and feedback in each of the two phases to achieve higher secret rates. Over a lossy network the advantage of separating the two phases and using feedback becomes clear.

Finally, we give outer bounds on the securely achievable rate and prove optimality of our scheme in some special cases.

\section{A. Related Work}

Secure network coding by Cai and Yeung [5], [6], [7] is a seminal work in the field of secret communication over networks. The secrecy capacity of an error-free network is established and linear achievability schemes are proposed. 
This work was followed by a number of alternative constructions and extensions [8], [9], [10], yet as far as we know ours is the first work that looks at network coding secrecy over erasure networks with feedback.

Secret communication over a noisy channel was investigated by Wyner [1] and the result was extended for networks by Cui [11]. Using a similar approach capacity results for broadcast erasure networks were derived [12]. However, none of these work take advantage of feedback and thus they offer any nonzero secrecy rate over a wiretapped channel only if the eavesdropper's observation is more noisy than the legitimate receiver's. By exploiting a limited rate feedback significantly higher rates are achievable [13], [14], [2]. These results were extended for a broadcast setting with multiple receivers [15], but the generalization for a multihop network is a new and challenging problem that we start investigating here.

\section{Network Model AND BACKGROUND}

\section{A. Network model}

Communication takes place over a network which is represented by a directed acyclic multigraph $\mathcal{G}(V, E)$, where $V$ is the set of network nodes and $E$ is the (multi-)set of edges. An eavesdropper Eve can select arbitrarily up to $z$ edges of the network to wiretap. $A \subseteq E$ denotes the subset of wiretapped edges, where $|A| \leq z$. We assume that $z$ is known as a design parameter, but $A$ is known only by the eavesdropper.

Every link $e=(u, v) \in E$ is an erasure channel with parameters $\delta, \delta_{E}$. The input alphabet of the channel is $\mathbb{F}_{q}^{L}$, length $L$ vectors of a finite field $\mathbb{F}_{q}$. We often call such vectors packets. The network node $v$ receives transmissions on $e$ with an erasure probability $\delta$, while in case $\delta_{E} \in A$, Eve receives packets sent over $e$ with an erasure probability $\delta_{E}$. All erasures are assumed to be independent. For a given channel $e=(u, v)$ let $X_{e}$ denote the channel input while $Y_{e}$ is the receptions of $v$ and $Z_{e}$ is the potential receptions of Eve. Then,

$$
\begin{aligned}
& \operatorname{Pr}\left\{Y_{e}=X_{e} \mid X_{e}\right\}=1-\delta, \quad \operatorname{Pr}\left\{Y_{e}=\perp \mid X_{e}\right\}=\delta \\
& \operatorname{Pr}\left\{Z_{e}=X_{e} \mid X_{e}\right\}=1-\delta_{E}, \quad \operatorname{Pr}\left\{Y_{e}=\perp \mid X_{e}\right\}=\delta_{E} \\
& \operatorname{Pr}\left\{Y_{e}, Z_{e} \mid X_{e}\right\}=\operatorname{Pr}\left\{Y_{e} \mid X_{e}\right\} \operatorname{Pr}\left\{Z_{e} \mid X_{e}\right\}
\end{aligned}
$$

where $\perp$ is the symbol of erasure.

After every transmission over a link $(u, v)$ node $v$ acknowledges its receptions (whether it received correctly or an erasure happened). The acknowledgments are available to all the nodes causally. We also assume that all the acknowledgments are public to the eavesdropper regardless of whether or not the given link is wiretapped.

A source node $s \in V$ has a message $W \in \mathbb{F}_{q}^{L N}$ to send securely to a set of destination nodes $D \subset V$. Source $s$ can further generate independent randomness $\Theta$. We assume that $\Theta$ is uniformly distributed and it can be generated without rate constraint. We will treat both $W$ and $\Theta$ as a row vector of packets. The length of $W$ is $N$, while the length of $\Theta$, i.e. the amount of randomness needed is a property of the communication scheme.

The multicast capacity of $\mathcal{G}$ with source $s$ and destination nodes $D$ is $h(1-\delta)$, where $h$ denotes the number of edges in the smallest value min-cut between $s$ and any $d \in D$. We introduce parameters $h=t+\ell$, where $t$ is the number of multihop paths between $s$ and $d$ while $\ell$ is the number of direct $s$ - $d$ links in the smallest value min-cut.

1) Notation: The set of incoming and outgoing edges of $v \in V$ are denoted by $I_{v}$ and $O_{v}$.

If $\mathcal{E} \subseteq E$ then $Y_{i, \mathcal{E}}$ denotes the set of received packets by the network nodes in the $i$ th time slot on the set of edges $\mathcal{E}$. Similarly for $\mathcal{V} \subseteq V$ the notation $Y_{i, \mathcal{V}}$ denotes the set of packets that the set of nodes $\mathcal{V}$ receives in the $i$ th time slot. In case there are parallel edges the notation $(u, v)$ means the set of edges starting from $u$ and ending at $v$.

We use $Y_{\mathcal{E}}^{i}$ and $Y_{\mathcal{V}}^{i}$ as a shorthand for $Y_{1, \mathcal{E}} \ldots Y_{i, \mathcal{E}}$ and $Y_{1, \mathcal{V}} \ldots Y_{i, \mathcal{V}}$. We apply the same notation for other vectors also. E.g. the source node transmits $X_{i, s}$ in the $i$ th time slot, while Eve's observation after $n$ time slots is $Z_{A}^{n}$.

$F_{i}$ denotes the acknowledgments of the $i$ th time slot.

To simplify notation, we express entropy and rate in terms of packets. This allows us to omit the constant factor $L \log q$, which is the size of one packet.

\section{B. Security and rate}

Definition 1: A secure scheme that uses every channel $e \in$ $E$ of the network $n$ times has parameters $(n, \epsilon, N)$. For all $1 \leq i \leq n$ it defines encoding maps $\phi_{i, e}$ for all $e \in E$ :

$$
\begin{aligned}
& X_{i,(s, v)}=\phi_{i,(s, v)}\left(W, \Theta, F^{i-1}\right) \\
& X_{i,(u, v)}=\phi_{i,(u, v)}\left(Y_{I_{u}}^{i-1}, F^{i-1}\right), \forall u \neq s .
\end{aligned}
$$

For all $d \in D$ it defines decoding maps $\psi_{d}$ such that

$$
\operatorname{Pr}\left\{\psi_{d}\left(Y_{I_{d}}^{n}\right) \neq W\right\}<\epsilon .
$$

Further, Eve learns negligible information about $W$ :

$$
I\left(W ; Z_{A}^{n}, F^{n}\right)<\epsilon .
$$

In all our cases we consider linear schemes and hence linear encoding and decoding maps. It follows that $X_{i, e}$ can be written in the following form:

$$
X_{i, e}=\left[\begin{array}{ll}
\Theta & W
\end{array}\right]\left[\begin{array}{l}
f_{i, e}^{\Theta T} \\
f_{i, e}^{W T}
\end{array}\right]
$$

where $f_{i, e}=\left[f_{i, e}^{\Theta} f_{i, e}^{W}\right]$ is the global encoding vector of edge $e$ in the $i$ th time slot. Here we explicitly separate in notation the coefficients of packets from $\Theta$ and packets from $W$.

Definition 2: A secure communication rate $\mathcal{R}$ is achievable over $\mathcal{G}$ if for any $\epsilon>0$ there exists a secure scheme with parameters $(n, \epsilon, N)$ such that

$$
\mathcal{R}-\epsilon<\frac{N}{n} \text {. }
$$

We call the highest achievable secure communication rate the secure capacity of the network. 


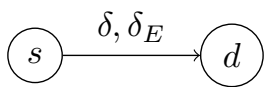

Fig. 1. Single link network

\section{Secure network coding over lossless networks}

In the special case of lossless channels $\delta=\delta_{E}=0$ our model becomes the same as seen in [5]. Here we shortly summarize the work of Cai and Yeung [5], [6]. A linear coding scheme known as the secure network coding scheme is proposed with - in our notation - parameters $\left(1, \epsilon,(h-z)^{+}\right)$. The scheme uses source randomness of size $z$ and ensures that all destination nodes receive both the message and the additional randomness. It is shown that the secure network coding scheme is optimal in terms of the achieved rate and it uses the minimum amount of additional randomness any optimal scheme might use.

Let us assume that Eve simply discards any packet that she receives more than once. We denote $z^{\prime} \leq z$ the number of innovative packets Eve observes. Then, we can write Eve's observation in the following form:

$$
Z_{A}=\left[\begin{array}{ll}
\Theta & W
\end{array}\right]\left[\begin{array}{c}
f_{A}^{\Theta T} \\
f_{A}^{W T}
\end{array}\right] .
$$

Here $f_{A}^{\Theta T}$ is a $z \times z^{\prime}$ matrix and $f_{A}^{W T}$ is a $(h-z) \times z^{\prime}$ matrix. The secure network coding scheme has the property that the matrix $f_{A}^{\Theta T}$ has rank $z^{\prime}$. Thus, $\Theta f_{A}^{\Theta T}$ is a set of $z^{\prime} \leq z$ independent uniform random packets, while $W f_{A}^{W T}$ is a set of $z^{\prime}$ linear combinations of the message packets, hence from Eve's perspective what she observes is some data $W f_{A}^{W T}$ encrypted using one time pad with key $\Theta f_{A}^{\Theta T}$.

One possible intuitive interpretation of these results is the following: to give perfect security against Eve who has access to at most $z$ innovative packets, we need to send $z$ packets of additional randomness and hence the secure capacity of the network is reduced by $z$ compared to its multicast capacity. We use this intuition when we design our scheme for lossy networks.

\section{Secure message sending over a single link}

Consider the simplest possible network shown in Fig. 1 consisting of a single channel with parameters $\delta, \delta_{E}$, and $z=1$.

1) Direct solution: One straightforward approach to deal with lossy networks is to use a forward error correction code at all the transmitting nodes and by this turn the noisy channels into error free channels. We can then treat the network a lossless network with links of capacity $(1-\delta)$ where we can apply the secure network coding scheme and achieve a rate $(h-z)(1-\delta)$. However, having a single wiretapped link we cannot achieve any secrecy this way. This approach is too pessimistic though, because it implicitly assumes that the eavesdropper does not experience any erasures after applying coding.
2) Exploiting Eve's erasures: As observed by Wyner [1] and applied for networks in [11], if $\delta_{E}>\delta$ despite of the error correction coding Eve still does not receive everything, which allows to achieve a secrecy rate $\left(\delta_{E}-\delta\right)^{+}$. Applying this result in a network, for $z \leq h$ a secrecy rate $(h-z)(1-$ $\delta)+z\left(\delta_{E}-\delta\right)^{+}$is achievable. In other words, as opposed to the direct solution a nonzero rate is potentially achieved also over the wiretapped edges [11].

3) Using feedback: By exploiting the acknowledging feedback we can do even better. Instead of a forward error correction code the source can send packets using an ARQ strategy, i.e. it repeats every packet until it is acknowledged. Using this strategy the next node experiences no erasures, while for Eve there is still a probability $\frac{\delta_{E}(1-\delta)}{1-\delta \delta_{E}}$ that she does not receive a certain packet. This situation is equivalent to a channel with capacity $(1-\delta)$ and parameters $\delta^{\prime}=0$, $\delta_{E}^{\prime}=\frac{\delta_{E}(1-\delta)}{1-\delta \delta_{E}}$. Hence, we can apply Wyner's scheme on this logical channel and achieve a secrecy rate $(1-\delta) \frac{\delta_{E}(1-\delta)}{1-\delta \delta_{E}}$.

4) Separation of phases: None of the previous strategies achieves capacity in this setting. An optimal two-phase coding scheme was proposed in [2], which we summarize here.

The scheme has a key generation phase and an encrypted message sending phase. In the first phase $s$ and $d$ agrees securely in a shared key, which key is used for encryption in the second phase.

a) Key generation phase: Source $s$ sends $n_{1}$ independent uniform random packets. For the moment, let us assume that $d$ receives exactly $(1-\delta) n_{1}$ packets, while Eve does not receive $\delta_{E}(1-\delta) n_{1}$ out of these. Let $M$ denote the $1 \times(1-\delta) n_{1}$ vector of the received packets. Let $H$ be a $(1-\delta) n_{1} \times \delta_{E}(1-\delta) n_{1}$ matrix, and let $H$ be a parity check matrix of an MDS code. Then both $s$ and $d$ can compute

$$
K=M H,
$$

where $K$ is a uniformly distributed random key of size $n_{1} \delta_{E}(1-\delta)$, for which $I\left(K F^{n_{1}} ; Z^{n_{1}}\right)=0$.

Of course, we have a probabilistic channel, hence we cannot assume that $d$ and Eve receive exactly as many packets as they are expected. Still a secret key can be established at the same rate with the following change of parameters.

Let $s$ send $n_{1}=n_{1}^{\prime}+n_{1}^{\prime \frac{3}{4}}$ independent and uniform random packets. If $d$ does not acknowledge $n_{1}^{\prime}(1-\delta)$ packets, then an error is declared. Let $M$ denote the first $n_{1}^{\prime}(1-\delta)$ packets that $d$ acknowledges. Let $k=\delta_{E}(1-\delta) n_{1}^{\prime}-n_{1}^{\prime \frac{3}{4}}$ and $H$ be a $\left(n_{1}^{\prime}(1-\delta) \times k\right)$ MDS parity check matrix. Both $s$ and $d$ calculates $K=M H$.

Theorem 1: [2] For any $\epsilon>0$ there exists a large enough $n_{1}$ for which $K$ is computable with an error probability smaller than $\epsilon$, further $K$ is uniformly distributed and

$$
\begin{aligned}
& I\left(K ; F^{n_{1}} Z^{n_{1}}\right)<\epsilon \\
& \frac{|K|}{n_{1}}>\delta_{E}(1-\delta)-\epsilon .
\end{aligned}
$$


In other words, the same key generation rate $\delta_{E}(1-\delta)$ is achievable as if $d$ and Eve received exactly as many packets as they are expected.

b) Encryption and encrypted message sending: Again, let us assume for a moment that $d$ and Eve receive as many packets as they are expected. Then let $N=|W|=n_{2}(1-\delta)$.

Given a secret key $K$ of size $|K|=n_{2}(1-\delta) \frac{1-\delta_{E}}{1-\delta \delta_{E}}$ established between $s$ and $d$, the encrypted message $W_{E}$ is calculated as follows:

$$
W_{E}=W+K G,
$$

where $G$ is a $(|K| \times N)$ and is a generator matrix of an MDS code. The packets of the encrypted message $W_{E}$ are then sent using ARQ. Note that Eve is expected to receive $n_{2}(1-$ $\delta) \frac{1-\delta_{E}}{1-\delta \delta_{E}}$ different packets of $W_{E}$, which is exactly the size of the key we use. Like in the case of secure network coding scheme Eve hence observes a one time pad encryption, from which the secrecy of the message follows.

In our probabilistic model we need to modify the parameters as follows:

$$
N=n_{2}(1-\delta)-n_{2}^{\frac{3}{4}} ; \quad|K|=n_{2}(1-\delta) \frac{1-\delta_{E}}{1-\delta \delta_{E}}+n_{2}^{\frac{3}{4}} .
$$

Given these, $G$ has to be of size $(|K| \times N)$.

Theorem 2: [2] Given a secret key $K$ as generated in the first phase, for any $\epsilon>0$ there exist large enough $n_{1}, n_{2}$ for which the probability that $d$ receives $W_{E}$ and can decode $W$ is larger than $1-\epsilon$, and

$$
\begin{aligned}
I\left(Z^{n} F^{n} ; W\right) & <\epsilon \\
\frac{N}{n} & >\delta_{E}(1-\delta) \frac{1-\delta \delta_{E}}{1-\delta \delta_{E}^{2}}-\epsilon .
\end{aligned}
$$

Further, no other scheme can achieve a rate larger than $\delta_{E}(1-\delta) \frac{1-\delta \delta_{E}}{1-\delta \delta_{E}^{2}}$.

Theorems 1-2 are stated in a slightly different form in [2] without explicitly separating the security properties of the two phases. Theorems 3-4 that we prove in Section IV generalize this result for a network setting.

\section{TWO-PHASE SECURE NETWORK CODING SCHEME}

In this section, we only examine lossless networks and simply make the point that the secure network coding scheme [5] - with a slight modification - can be cast as a two-phase scheme. We show that the modification does not effect the achieved rate, hence the two phase secure network coding scheme is also optimal. That is, we provide a new, alternative achievability scheme achieving the secure capacity of a lossless network. In Section IV we will derive a unified achievability scheme that will accept as special cases the achievability scheme we provide next for lossless networks, as well as the achievability scheme for the single channel erasure network we described in Section II-D.

\section{A. Example}

For simplicity in this example we assume unicast traffic. Consider the following simple network (Fig. 2). Source $s$ and destination $d$ are connected through two parallel unit capacity
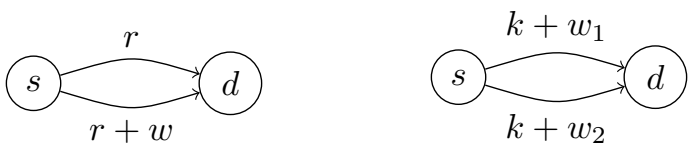

Fig. 2. Secure network coding

Fig. 3. Coding with shared key

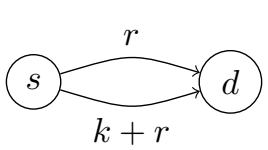

(a) First time slot

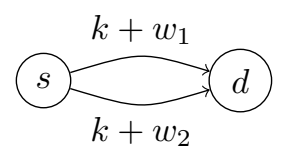

(b) Second time slot
Fig. 4. Two-phase scheme example

lossless links out of which any one is being wiretapped by Eve ( $h=2, z=1)$. The secrecy capacity of the network is 1 , hence $s$ can send securely a unit size message $w$. To apply a secure network coding scheme it generates a unit size randomness $r$. As shown in the figure, on one of the links $s$ sends $r$ while on the other link it sends $r+w$. The eavesdropper either sees $r$ or $r+w$, in either case no information about $w$ is leaked.

Assume now, that $s$ and $d$ already share a unit size random key $k$, which is not known by Eve. Then, as shown in Fig. 3 $s$ can securely send two unit size messages $w_{1}$ and $w_{2}$ using $k$ for encryption on both links. Hence, in this case a unit size shared key allows us to exploit the min-cut capacity of the network. One might ask, how $s$ and $d$ can set up a shared key. The secure network coding scheme offers a way to send any message $w$ securely to $d$, this message can equally well be a key $k$. Consider the example in Fig. 4, where in two time slots two messages are sent securely to $d$. In the first slot a key is set up, while in the second slot this key is used for encryption. Note that the achieved rate is 1 , the same as what the secure network coding scheme achieves. Also the amount of additional randomness remains the same.

To avoid confusion we note here that the randomness used in the secure network coding scheme is often called a key. Indeed, this randomness is used to encrypt a message, however this randomness is not known by $d$ at the moment of encryption. Further, this randomness does not necessarily remain secret from Eve. E.g. in our example in Fig. 2 if Eve selects the top link to wiretap, she learns $r$. To distinguish source randomness from keys we call a key a shared randomness between $s$ and $d$ which randomness is secret from Eve at the moment of encryption. We also have the property that the key remains secret from Eve given the message is uniformly distributed.

\section{B. Scheme description}

The properties that we have seen through our example can be generalized as follows. We call the scheme described below the two-phase secure network coding scheme. As opposed to the secure network coding scheme our scheme uses every link $n=n_{1}+n_{2}$ times, where $n_{1}$ and $n_{2}$ are the number of time slots used for the two phases respectively. We use the secure network coding scheme as a building block, 
we select one such code at the outset and then in each of the $n$ time slots we use the same code on different inputs. Hence, we have that $f_{i, e}=f_{j, e}=f_{e}, \forall i, j$.

In our scheme the size of our message is $N=n_{2} h$. To securely send a message of this size, we need a shared key $K$ of size $n_{2} z$ between $s$ and the destination nodes.

1. Key generation: The sender generates a uniformly random $K$ of size $n_{2} z$. It also generates additional randomness $\Theta$ of size $n_{2} \frac{z^{2}}{h-z}$. The key generation phase consists of $n_{1}=n_{2} \frac{z}{h-z}$ time slots, in each slot $s$ securely sends $h-z$ packets from $K$. On edge $e$ in the $i$ th slot we send thus

$$
[\Theta(i) \quad K(i)] f_{e},
$$

where $K(i)$ is the $i$ th $h-z$ length fraction of $K: K(i)=$ $K_{(i-1)(h-z)+1 \ldots i(h-z)}$. Similarly, $\Theta(i)$ is the $i$ th $z$ length fraction of $\Theta: \Theta(i)=\Theta_{(i-1) z+1 \ldots i z}$.

2. Encrypted message sending: In the second phase we use $K$ for encryption and in each slot $h$ message packets are sent securely. We use again the same secure network code $n_{2}$ times. We denote $W(i)$ the first $h-z$ elements of the $i$ th $h$ length fraction of $W$ and $W^{\prime}(i)$ the last $z$ elements of the same fraction. On edge $e$ in the $i$ th slot of the second phase we then send

$$
\left[W^{\prime}(i)+K(i) \quad W(i)\right] f_{e} .
$$

It directly follows from the properties of the secure network coding scheme that all destination nodes know $K$ and hence can decode $W$.

Building on the security of the secure network code we use we show that the scheme is secure. We delegate the proof of security to to our technical report [16].

Achieved rate: Our scheme conveys a message of size $n_{2} h$ using $n_{1}+n_{2}$ transmissions, thus our rate is

$$
\mathcal{R}=\frac{n_{2} h}{n_{1}+n_{2}}=\frac{n_{2} h}{n_{2} \frac{z}{h-z}+n_{2}}=h-z,
$$

which is the same as the rate of the secure network coding scheme. We further note that the amount of randomness we use is $|K|+|\Theta|=n_{2} \frac{h z}{h-z}$, which is also the same as the secure network coding scheme uses to securely send a message of size $n_{2} h$. By selecting $n_{2}=h-z$ the rate $h-z$ is achieved in a finite block length.

\section{Discussion}

The two-phase secure network coding scheme gives us a way to isolate two different problems, the key generation and the message communication problem. We have to note that our security argument holds for any secure key generation phase. In the case of lossless networks the separation does not make any difference in the achieved rates, since the rate of key generation is the same as the achievable rate of secret message sending. However, in some cases this might not hold and a higher key generation rate is possible. In those cases designing the two phases separately results in an improved secure communication rate.

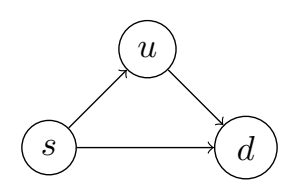

Fig. 5. Example network

\section{OUR ACHIEVABILITY SCHEME}

We first describe our scheme for a single receiver node $D=d$, then in Section IV-C we generalize our description for multicast. We also assume here that $z \leq h$. This assumption was necessary in case of an error free network to achieve any nonzero rate securely, however in an erasure network we can achieve some rate even if $z>h$. We discuss this case in a subsequent section.

\section{A. Example}

Before a detailed description, we explain ideas through the example network in Fig. 5. Let $z=1$. For simplicity, in the example when we calculate the number of received packets we work with expected values instead of random variables.

1) Key generation: Source $s$ sends independent random packets over all its outgoing links. Both the destination node $d$ and the intermediate node $u$ receive $n_{1}(1-\delta)$ packets. On the link between $u$ and $d$ the packets that $u$ received are then sent to $d$ using ARQ. To complete this task $u$ needs $n_{1}$ transmissions.

The achievable key rate corresponds to the number of packets that $d$ receives but Eve does not. Eve has three possible choices to select a wiretapped link, and when generating the key we need to consider her worst-case selection.

Case 1: Eve selects the $s$ - $d$ link. In this case the number of packets that both $d$ and Eve receive is $n_{1}(1-\delta)\left(1-\delta_{E}\right)$.

Case 2: Eve selects the $u$-d link. Since $d$ eventually receives every packet that $u$ has and every packet is repeated potentially several times, the probability that Eve overhears a certain packet of $d$ is increased to $\frac{1-\delta_{E}}{1-\delta \delta_{E}}$. Node $u$ sends $n_{1}(1-\delta)$ different packets, hence Eve has $n_{1}(1-\delta) \frac{1-\delta_{E}}{1-\delta \delta_{E}}$ packets in common with $d$.

Case 3: Eve selects the $s$ - $u$ link. We know that all the packets that $u$ receives $d$ also receives. Eve and $u$ have $n_{1}(1-$ $\delta)\left(1-\delta_{E}\right)$ packets that they both receive, we get the same result as in the first case.

We conclude that Eve's best choice (from her perspective) is the $u-d$ link. Destination $d$ has

$$
|K|=2 n_{1}(1-\delta)-n_{1}(1-\delta) \frac{1-\delta_{E}}{1-\delta \delta_{E}}
$$

packets not received by Eve, hence a key rate of $1-\delta+$ $\frac{(1-\delta)^{2} \delta_{E}}{1-\delta \delta_{E}}$ is achievable.

2) Encrypted message transmission: There are two edge disjoint paths between $s$ and $d$. Let $n_{2}$ be the number of transmissions in the second phase. The message is encrypted in the form that we have already seen: $W_{E}=W+K G$, where $K$ is the key and $G$ is an MDS generator matrix. $W_{E}$ is split into two parts and each half of the message is assigned to one of the paths. The message packets are then forwarded towards $d$ using ARQ on each link. 
The size of the key $K$ we use has to equal the number of packets Eve receives in the second phase. In this case, the MDS property of $G$ ensures that Eve receives every packet with an independent linear combination of $K$, thus the security of the scheme follows.

Since the same forwarding strategy is applied on each link, regardless of which link Eve selects, she receives a certain packet with probability $\frac{1-\delta_{E}}{1-\delta \delta_{E}}$, thus she receives overall $n_{2}(1-\delta) \frac{1-\delta_{E}}{1-\delta \delta_{E}}$ different packets. Hence $n_{1}$ and $n_{2}$ are chosen such that $|K|=n_{2}(1-\delta) \frac{1-\delta_{E}}{1-\delta \delta_{E}}$.

\section{B. Algorithm}

As a first step we select $h$ edge disjoint paths between $s$ and $d$. We ignore all other edges of $\mathcal{G}$. The example in the previous section suggests that the achievable rate depends not only on $h, z, \delta$ and $\delta_{E}$, but also on the number of direct $s$ - $d$ links. Let $h=\ell+t$, where $\ell$ denotes the number of direct $s$ - $d$ links and $t$ denotes the number of multihop paths.

1) Key generation: We define

$$
\begin{aligned}
n_{1}^{\prime}= & n_{1}-n_{1}^{\frac{3}{4}} \\
\zeta_{1}= & n_{1}^{\prime}(z-t)^{+}(1-\delta)\left(1-\delta_{E}\right) \\
& +n_{1}^{\prime} \min \{z, t\}(1-\delta) \frac{1-\delta_{E}}{1-\delta \delta_{E}} \\
|K|= & h n_{1}^{\prime}(1-\delta)-\zeta_{1}-n_{1}^{\prime \frac{3}{4}} .
\end{aligned}
$$

Source $s$ sends at most $n_{1}$ random packets on all its $h$ outgoing edges. It stops transmission on each link as soon as $n_{1}^{\prime}(1-\delta)$ packets are acknowledged on the given link. Intermediate nodes on each path forward the $n_{1}^{\prime}(1-\delta)$ packets that they receive to the next node on the path towards $d$ using ARQ.

If $d$ does not receive $h n_{1}^{\prime}(1-\delta)$ packets, then an error is declared. Otherwise, let $M$ denote the vector of all the packets that $d$ receives. Both $s$ and $d$ compute

$$
K=M H,
$$

where $H$ is a $\left(h n_{1}^{\prime}(1-\delta) \times|K|\right)$ matrix and it is a parity check matrix of an MDS code.

2) Encryption and message sending: We find $N, n_{2}$ and $n_{2}^{\prime}$ such that

$$
\begin{array}{ll}
\zeta_{2}=n_{2}^{\prime} z(1-\delta) \frac{1-\delta_{E}}{1-\delta \delta_{E}} ; & |K|=\zeta_{2}+n_{2}^{\prime \frac{3}{4}} \\
n_{2}^{\prime}=n_{2}-n_{2}^{\frac{3}{4}} ; & N=h n_{2}^{\prime}(1-\delta)
\end{array}
$$

The encrypted message $W_{E}$ is computed as

$$
W_{E}=W+K G
$$

where $K$ is the key from the first phase and $G$ is a $(|K| \times N)$ matrix and it is a generator of an MDS code.

We assign $n_{2}^{\prime}(1-\delta)$ packets to each of our paths. These packets are then forwarded on their assigned path to $d$ using ARQ over each link. If $d$ does not receive all the packets of $W_{E}$ after $n_{2}$ transmissions, then an error is declared.

\section{Multicast}

In this section we present our scheme for the multicast problem, where there are more than one destination nodes and all of them have to receive the same message securely. Compared to the unicast scheme only a few modifications are needed. To avoid repetition, below we highlight only the differences.

Instead of $h$ edge disjoint paths, first we need to find a network code for multicasting at rate $(1-\delta) h$. Again, we can ignore all edges that are not used by the network code.

In the key generation phase we need the following modification. Instead of sending new random packets on the outgoing edges, $s$ selects in advance $n_{1}^{\prime} h(1-\delta)$ random packets that are sent reliably to all destination nodes using ARQ on each link and applying the network code that we have chosen. The same network code is used in each time slot. This ensures that all $d \in D$ receive the same set of packets and hence they all can compute the same key. According to this we modify parameter $\zeta_{1}$ :

$$
\zeta_{1}=n_{1}^{\prime} z(1-\delta) \frac{1-\delta_{E}}{1-\delta \delta_{E}} .
$$

Note that this change implies a change of parameters $|K|, n_{2}, n_{2}^{\prime}, \zeta_{2}$ and $N$, however all formulas remain the same as defined for unicast.

In the second phase the only difference is that instead of forwarding through $h$ edge-disjoint paths we use the network code (together with ARQ) to reliably send the encrypted packets to all destinations.

Another modification is needed in the selection of matrices $H$ and $G$. Note that in the unicast case intermediate network nodes do not perform any coding, hence Eve might only receive packets that $s$ produces. This property enables to code only at the source using any $H$ and $G$ matrices that have MDS property. In case of multicast, intermediate nodes might produce new linear combinations, hence Eve might receive combined packets as well.

As for matrix $H$, consider the $h n_{1}^{\prime}(1-\delta)$ packets that $s$ sends in the key generation phase and all their different linear combinations that the prescribed network code produces. Let $f_{A}^{n_{1}}$ denote a coefficient matrix of size $h n_{1}^{\prime}(1-\delta) \times h n_{1}^{\prime}(1-$ $\delta)-|K|$ that describes a $h n_{1}^{\prime}(1-\delta)-|K|$ size subset of these packets. This subset corresponds to a set of packets that Eve might receive. We will see during the analysis that the probability that Eve receives a larger subset of packets is negligible. We select $H$ such that $\left[\begin{array}{ll}H & f_{A}^{n_{1}}\end{array}\right]$ is a full rank (in fact invertible) matrix for all possible $f_{A}^{n_{1}}$. This property ensures the security of the generated keys. Later we show that such $H$ exists and that generated keys are secure.

As for matrix $G$, we consider a $|K|$ size subset of the different encoded packets that Eve might receive during the second phase. Let $f_{A}^{n_{2} K}$ denote the $|K| \times|K|$ coefficient matrix of Eve's possible receptions that contain the coefficients of packets from $K$. We select $G$ such that all possible such $f_{A}^{n_{2} K}$ matrix is invertible. As shown in our analysis this property ensures security of the message. 
The existence of such matrices $H$ and $G$ is a direct consequence of known results. The conditions we pose for $H$ and $G$ are the same conditions that need to be satisfied when finding a secure network code. In fact $G$ itself gives a secure network code in a network with parameters $h=|E| n_{2}^{\prime}(1-\delta), z=|K|$. Our condition for $H$ can be satisfied as proved by Lemma 3 of [5]. Hence, the existence of such $H$ and $G$ matrices over a sufficiently large field is shown by Lemma 3 and Theorem 2 from [5]. A worst case estimate for the required field size is $q \leq \max \left\{\left(\begin{array}{c}\left(\begin{array}{c}|E| \\ z\end{array}\right) n_{1}^{\prime} \\ n_{1}^{\prime}(1-\delta)-|K|\end{array}\right),\left(\begin{array}{c}\left(\begin{array}{c}|E| \\ z\end{array}\right) n_{2}^{\prime}(1-\delta) \\ |K|\end{array}\right)\right\}$, however any construction proposed for secure network coding (e.g. [17], [18]) can be used to find $H$ and $G$.

\section{Analysis}

In case of unicast, the key generation phase achieves a key rate

$$
\begin{aligned}
\kappa= & h(1-\delta)-(z-t)^{+}(1-\delta)\left(1-\delta_{E}\right) \\
& -\min \{z, t\}(1-\delta) \frac{1-\delta_{E}}{1-\delta \delta_{E}}
\end{aligned}
$$

while in case of multicast a key rate

$$
\kappa=h(1-\delta)-z(1-\delta) \frac{1-\delta_{E}}{1-\delta \delta_{E}}
$$

is achieved according to the following theorem.

Theorem 3: For any $\epsilon>0$ there exists a large enough $n_{1}$ such that the key generation runs without error with probability at least $1-\epsilon, K$ is uniformly distributed, and the following inequalities hold for any $A \subseteq E,|A|=z \leq h$,

$$
\begin{aligned}
& I\left(Z_{A}^{n_{1}}, F^{n_{1}} ; K\right)<\epsilon, \text { and } \\
& \frac{|K|}{n_{1}}>\kappa-\epsilon,
\end{aligned}
$$

where in case of unicast $\kappa$ is as defined by (2), while in case of multicast (3) applies for $\kappa$.

The scheme is secure and achieves a rate $\mathcal{R}$ as given by the next theorem.

Theorem 4: For any $\epsilon>0$ there exists a large enough $n=n_{1}+n_{2}$ such that the above scheme is secure as defined in Definition 1 and achieves a rate

$$
\mathcal{R}=\frac{h}{z \frac{1-\delta_{E}}{\kappa\left(1-\delta \delta_{E}\right)}+\frac{1}{1-\delta}},
$$

where $\kappa$ is as defined by (2) for unicast and by (3) for multicast.

We provide the proofs of Theorems 3-4 in [16] (available online).

With $\delta=\delta_{E}=0$ we see that $\kappa=h-z$ and $\mathcal{R}=h-z$, hence in this special case the scheme achieves the same rate as the secure network coding scheme. Also, for $h=z=\ell=$ 1 we have $\kappa=\delta_{E}(1-\delta)$ and get back $\mathcal{R}=\delta_{E}(1-\delta) \frac{1-\delta \delta_{E}}{1-\delta \delta_{E}^{2}}$, the optimal rate of a single channel network.

Beside these two, we show optimality for some further cases, see Section V, Theorems 5-6 for outer bounds and Corollary 1 for the optimality result.

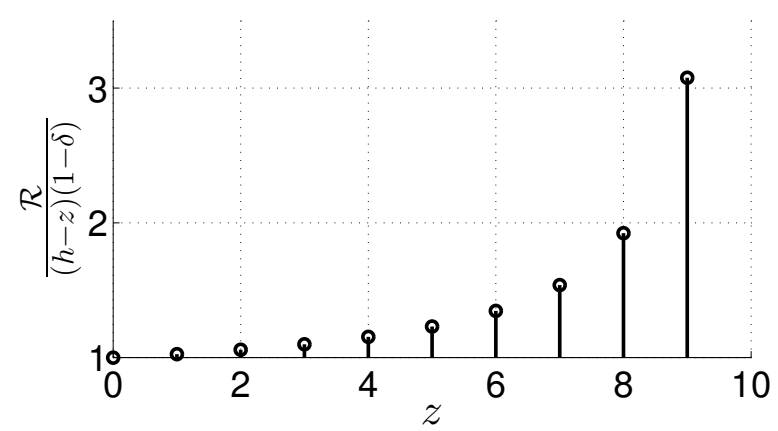

Fig. 6. Advantage of using feedback as a function of the number of eavesdropped edges $z$, when $h=t=10, \delta=\delta_{E}=0.3$. In this case multicast and unicast rates are the same.

1) Qualitative comparison: The direct application of secure network coding in an erasure network - as we described in Section II-D - allows a rate $(h-z)(1-\delta)$. In case $\delta \geq \delta_{E}$, taking into account Eve's erasures, but not using feedback does not allow any better rates [11]. The advantage of exploiting feedback is twofold. First, it allows a higher key generation rate $\kappa \geq(h-z)(1-\delta)$. Second, it allows to reduce the size of the key we need in the second phase from $n_{2} z(1-\delta)$ to $n_{2} z(1-\delta) \frac{1-\delta_{E}}{1-\delta \delta_{E}}$. In this section we illustrate qualitatively how large this advantage is.

One can immediately see that the larger $\delta_{E}$ and $z$ are the larger the advantage of using feedback is. In particular if $z=h$ our scheme still achieves a nonzero rate, which is not possible without feedback (assuming $\delta_{E} \leq \delta$ ).

In our example we consider the case when $\delta=\delta_{E}$. In this case the best achievable rate without feedback is $(h-z)(1-$ $\delta)$. We consider a network with parameters $h=t=10$ and $\delta=0.3$. We plot in Fig. 6 the advantage of our scheme as the ratio between $\mathcal{R}$ and $(h-z)(1-\delta)$. We see that in this case our scheme achieves a rate up to 3 times higher than the scheme without feedback. With the increase of the network size or $\delta=\delta_{E}$ the advantage becomes even larger. Note that we have selected the parameter values for the example such that there is no difference between the unicast and the multicast rate of our scheme.

\section{OUTER BOUNDS}

In this section we provide outer bounds on the securely achievable rates in our network model. In some cases (see Corollary 1) the outer bound and the rate achieved by our scheme match, thus the presented scheme is optimal. For other cases we perform numerical evaluations to compare the achieved rates with the upper bound.

When deriving our upper bounds we make the following two assumptions which can only increase the achievable rates: (a) The set of eavesdropped edges are known, hence we restrict Eve to one particular selection of edges. (b) The state of the eavesdropper's channel is also known to every node in the network. In particular we give two bounds. The first bound is valid for any network and depends on $h$ and $z$, while the other is valid for networks where $O_{s}=I_{d}=h$ and beside $h$ and $z$ parameter $t$ also plays a role. 


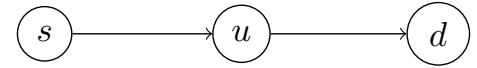

Fig. 7. Two-hop line network

Theorem 5: Assuming $z \leq h$, for the securely achievable rate over $\mathcal{G}$ it holds that

$$
\mathcal{R} \leq(1-\delta)(h-z)+z \delta_{E}(1-\delta) \frac{1-\delta \delta_{E}}{1-\delta \delta_{E}^{2}} .
$$

We note here that when $z>h$ we can substitute $z=h$ to get a valid upper bound for all cases. (The secure capacity cannot decrease by decreasing $z$.)

Theorem 6: Assuming $O_{s}=I_{d}=h$, for the securely achievable rate over $\mathcal{G}$ it holds that

$$
\mathcal{R} \leq(1-\delta) h-\min \{t, z\} \frac{\left(1-\delta_{E}\right)(1-\delta)}{1-\delta \delta_{E}} .
$$

We provide proofs of Theorems 5-6 in [16]. As a corollary of Theorems 5-6 we have the following optimality result.

Corollary 1: Our scheme presented in Section IV achieves secure capacity in the following cases:

1) $h=\ell=z$,

2) $O_{s}=I_{d}=h$ and $t \geq z$,

3) $\delta_{E}=0$ or $\delta_{E}=1$.

\section{DISCUSSION}

\section{A. Code constructions}

Theorems 3-4 show the achievability of the claimed secret message rate, however no practical code constructions are given. In case of a unicast problem we need codes with MDS property, for which efficient constructions exist. In case of a multicast problem the codes need to satisfy more constraints, which makes the associated coding problem significantly harder. Giving a practical algorithm for finding codes with the required property for the multicast problem remains an open question.

\section{B. Extension for $z>h$}

Assume $z=2$ and consider the two-hop line network shown in Fig. 7. Against this stronger Eve, we can run our scheme as presented in Section IV, but with different parameters. We need to calculate how many packets Eve might receive in each phase. We give the calculation in expectation.

In the message sending phase Eve has two independent chances to overhear a certain packet, on each link she receives a given packet with probability $\frac{1-\delta_{E}}{1-\delta \delta_{E}}$, hence the number of different packets she receives (in expectation) is:

$$
n_{2} \frac{1-\delta_{E}}{1-\delta \delta_{E}}+n_{2} \frac{1-\delta_{E}}{1-\delta \delta_{E}}\left(1-\frac{1-\delta_{E}}{1-\delta \delta_{E}}\right) .
$$

In the key generation phase she gets $n_{1}(1-\delta)\left(1-\delta_{E}\right)$ packets in common with $u$ on the first link, while she receives a packet with probability $\frac{1-\delta_{E}}{1-\delta \delta_{E}}$ on the second link, hence she is expected to get

$$
n_{1}(1-\delta)\left(1-\delta_{E}\right)+n_{1}\left(1-(1-\delta)\left(1-\delta_{E}\right)\right) \frac{1-\delta_{E}}{1-\delta \delta_{E}}
$$

packets in common with $d$, which allows a key rate

$$
\kappa=\delta_{E}(1-\delta)-\left(1-(1-\delta)\left(1-\delta_{E}\right)\right) \frac{1-\delta_{E}}{1-\delta \delta_{E}} .
$$

To calculate the achievable rate $\frac{(1-\delta) n_{2}}{n_{1}+n_{2}}$ we need to consider $n_{1}$ and $n_{2}$ such that

$$
n_{1} \kappa=n_{2} \frac{1-\delta_{E}}{1-\delta \delta_{E}}+n_{2} \frac{1-\delta_{E}}{1-\delta \delta_{E}}\left(1-\frac{1-\delta_{E}}{1-\delta \delta_{E}}\right) .
$$

Note that for any given network and any given set of wiretapped edges a similar analysis is feasible. After investigating all the $\left(\begin{array}{c}|E| \\ z\end{array}\right)$ possible sets of wiretapped edges, we can design our code such that it provides secrecy against all possible eavesdropped sets. However, the worst-case selection of eavesdropped edges and thus the actual rates achieved highly depends on the topology of our network.

\section{REFERENCES}

[1] A. D. Wyner, "The wire-tap channel," The Bell system Technical Journal, vol. 54, no. 8, pp. 1355-1387, 1975.

[2] L. Czap, V. Prabhakaran, C. Fragouli, and S. Diggavi, "Secret message capacity of erasure broadcast channels with feedback," in Information Theory Workshop (ITW), 2011, pp. 65-69.

[3] M. Langberg and M. Médard, "On the multiple unicast network coding, conjecture," in 47th Annual Allerton Conference on Соттиnication, Control, and Computing. IEEE, 2009, pp. 222-227.

[4] W. Huang, T. Ho, M. Langberg, and J. Kliewer, "On secure network coding with uniform wiretap sets," in International Symposium on Network Coding (NetCod). IEEE, 2013.

[5] N. Cai and R. Yeung, "Secure network coding on a wiretap network," IEEE Transactions on Information Theory, vol. 57, no. 1, pp. 424435, 2011.

[6] - "Secure network coding," in International Symposium on Information Theory (ISIT). IEEE, 2005, p. 323.

[7] R. W. Yeung and N. Cai, "On the optimality of a construction of secure network codes." in IEEE International Symposium on Information Theory (ISIT). IEEE, 2008, pp. 166-170.

[8] K. Jain, "Security based on network topology against the wiretapping attack," Wireless Communications, IEEE, vol. 11, no. 1, pp. 68-71, 2004.

[9] J. Feldman, T. Malkin, C. Stein, and R. Servedio, "On the capacity of secure network coding," in Allerton Conference on Communication, Control, and Computing, 2004.

[10] S. Rouayheb and E. Soljanin, "On wiretap networks II," in International Symposium on Information Theory (ISIT), 2007.

[11] T. Cui, "Coding for wireless broadcast and network secrecy," Ph.D. dissertation, California Institute of Technology, 2010.

[12] A. Mills, B. Smith, T. Clancy, E. Soljanin, and S. Vishwanath, "On secure communication over wireless erasure networks," in IEEE International Symposium on Information Theory (ISIT), 2008, pp. 161165.

[13] U. Maurer, "Secret key agreement by public discussion from common information," IEEE Transactions on Information Theory, vol. 39, no. 3 , pp. 733-742, May 1993.

[14] M. Jafari Siavoshani, S. Diggavi, C. Fragouli, U. K. Pulleti, and K. Argyraki, "Group Secret Key Generation over Broadcast Erasure Channels," in Asilomar Conference on Signals, Systems, and Computers, 2010, pp. 719-723.

[15] L. Czap, V. Prabhakaran, S. Diggavi, and C. Fragouli, "Broadcasting private messages securely," in International Symposium on Information Theory (ISIT). IEEE, 2012, pp. 428-432.

[16] —, "Secure network coding with erasures and feedback," Tech Rep. [Online]. Available: https://arni.epfl.ch/bibliography/attachments/ single/138

[17] S. E. Rouayheb, E. Soljanin, and A. Sprintson, "Secure network coding for wiretap networks of type II," IEEE Transactions on Information Theory, vol. 58, no. 3, pp. 1361-1371, 2012.

[18] D. Silva and F. R. Kschischang, "Security for wiretap networks via rank-metric codes," in IEEE International Symposium on Information Theory (ISIT). IEEE, 2008, pp. 176-180. 